\title{
Correção cirúrgica dos tumores primários do coração
}

\author{
Camilo ABDULMASSIH NETO*, Pedro R. SALERNO*, Jarbas J. DINKHUYSEN*, Paulo CHACCUR*, \\ Antoninho S. ARNONI*, M.M.B. ZAMORANO*, Adib D. JATENE ${ }^{\star \star}$, Luiz Carlos Bento de SOUZA*, Paulo \\ P. PAULISTA*
}

ABDULMASSIH, NETO, C.; SALERNO, P.R.; DINKHUYSEN, J.J.; CHACCUR, P.; ARNONI, A.S.; ZAMORANO, M.M.B.; JATENE, A.D.; SOUZA, L.C.B.; PAULISTA, P.P. - Correçāo cirúrgica dos tumores primários do coração. Rev. Bras. Cir. Cardiovasc., 7(2):145-152, 1992.

RESUMO: No período de 1962 a 1991, no Instituto Dante Pazzanese de Cardiologia, foram tratados cirurgicamente 52 pacientes portadores de tumores primários do coraçāo. Dentre os tumores benignos, 43 eram mixomas, 5 fibromas, 1 fibroelastoma papilar, 1 rabdomioma e apenas 2 malignos (rabdomiossarcomas). A idade média foi de 41,1 anos; 33 pacientes eram do sexo feminino e 19 do masculino; $53,84 \%$ apresentaramse com insuficiência cardíaca congestiva, $19,2 \% \mathrm{com}$ dor precordial e/ou palpitaçōes e $17,3 \% \mathrm{com}$ taquiarritmias. A ressecçāo completa do tumor foi possível em 50 pacientes e os 2 com tumores malignos foram apenas submetidos a biopsia. Todos os pacientes sobreviveram à cirurgia; entretanto, 3 pacientes foram a óbito no período hospitalar. Um paciente com mixoma apresentou recorrência três anos após e foi reoperado com sucesso. Temos em acompanhamento tardio 20 pacientes, que se encontram em classe funcional I (NYHA) es resultados sảo bastante satisfatórios.

DESCRITORES: tumores do coraçāo, cirurgia.

\section{INTRODUÇĀO}

Os tumores primários do coraçāo e do pericárdio são bastante raros; representam $5 \%$ a $10 \%$ das neoplasias do órgāo e de seu anexo, com uma incidência de $0,0001 \%$ a $0,05 \%$ nas necropsias ${ }^{31}$.

Cerca de $75 \%$ das neoplasias primárias do coraçāo são benignas ${ }^{18}$. Por outro lado, as metástases são 10 a 40 vezes mais freqüentes e têm sido encontradas em $21 \%$ das necropsias de pacientes com câncer ${ }^{4}$.

As neoplasias cardíacas podem ser classificadas de acordo com a sua localizaçāo, ou seu aspecto histológico. No pericárdio, os tumores primários, benignos ou malignos, têm a mesma prevalência. No coração, por ordem decrescente de ocor- rência, os tumores benignos são: rabdomiomas, lipomas, fibromas e teratomas ${ }^{18}$.

Nos adultos, aproximadamente $25 \%$ dos tumores são malignos e correspondem: $33 \%$ de angiossarcomas, $20 \%$ de rabdomiossarcomas, $15 \%$ de mesoteliomas e $10 \%$ de fibrossarcomas ${ }^{24}$.

Os tumores cardíacos primários da infância diferem do adulto, são raros e não ultrapassam a $10 \%$ de todos os tumores nesta faixa etária. Os mais comuns săo os rabdomiomas, os fibromas e os teratomas ${ }^{28}$.

Este trabalho procura mostrar a experiência do Instituto Dante Pazzanese de Cardiologia no manuseio cirúrgico desses tumores benignos e malignos.

Trabalho realizado no Instituto Dante Pazzanese de Cardiologia. Sáo Paulo, SP, Brasil.

Apresentado ao $19^{\circ}$ Congresso Nacional de Cirurgia Cardíaca. São Paulo, 7 a 9 de maio, 1992.

* Do Instituto Dante Pazzanese de Cardiologia.

* Do Hospital do Coração da Associação do Sanatório Sírio.

Endereço para separatas: Camilo Abdulmassih Neto. Av. Dr. Dante Pazzanese, 500. Ibirapuera, 04012 São Paulo, SP, Brasil. 
ABDULMASSIH, NETO, C.; SALERNO, P.R.; DINKHUYSEN, J.J.; CHACCUR, P.; ARNONI, A.S.; ZAMORANO, M.M.B.; JATENE, A.D.; SOUZA, L.C.B.; PAULISTA, P.P. - Correção cirúrgica dos tumores primários do coraçāo. Rev. Bras. Cir. Cardiovasc., 7(2):145-152, 1992.

\section{CASUÍSTICA E MÉTODOS}

Foram tratados cirurgicamente 52 pacientes portadores de tumores primários do coração, no período de janeiro de 1962 a dezembro de 1991. O sexo feminino predominou em $63,4 \%$ dos casos ( 33 pacientes). A idade variou de 15 dias e 68 anos (média 40,1 $\pm 17,8$ anos).

Todos os pacientes eram sintomáticos; $53,84 \%$ encontravam-se em classe funcional III ou IV, $19,2 \%$ apresentavam queixa de desconforto precordial e/ ou palpitaçōes e em $17,3 \%$ foram detectadas arritmias cardiacas do tipo taquiarritmias. 0 diagnóstico foi realizado através de estudo hemodinâmico e/ou ecocardiograma em $90,3 \%$ dos casos, sendo apenas o ecocardiograma em $9,6 \%$. Em um dos pacientes foi utilizada a ressonância magnética como método complementar de diagnóstico. O eletrocardiograma não se mostrou característico para o diagnóstico da doença.

A via de acesso utilizada para a abordagem cirúrgica do tumor foi a toracotomia mediana em $44,2 \%$ e lateral em $55,7 \%$ dos pacientes. Cardioplegia cristalóide a $4^{\circ} \mathrm{C}$ associada a hipotermia moderada foi utilizada em $17,3 \%$ dos casos. Em todos os outros pacientes empregou-se hipotermia moderada com pinçamento aórtico intermitente como método de proteção miocárdica. Em $5,7 \%$ dos pacientes houve necessidade de colocação de retalho de pericárdio bovino no septo interatrial.

O mixoma foi o tumor cardíaco mais freqüente. Encontrado em $43(82,6 \%)$ pacientes, sendo 37 $(86 \%)$ em átrio esquerdo (AE) e $6(14 \%)$ no átrio direito (AD). Associado aos mixomas de AE encontrou-se deformidade do aparelho valvar mitral em 3 $(8,1 \%)$ pacientes, devido a infiltraçāo mixomatosa, sendo necessário realizar, em 1 , a cerclagem do anel mitral. Quatro $(10,8 \%)$ pacientes apresentavam trombo no interior do átrio esquerdo e $1(2,7 \%)$ dissecçāo crônica de aorta, que foi tratada com cola biológica. Duas pacientes foram operadas durante á gestação, uma na $1^{\text {a }}$ semana e a outra na $28^{a}$, evoluindo para parto normal a termo, sem intercorrências e gerando crianças normais.

Entre os portadores de mixoma de $A D, 2(33,3 \%)$ pacientes foram submetidos a troca valvar em posição tricúspide. Em 1 foi colocada prótese de StarrEdwards, devido à não existência, na época (década de 60), de prótese biológica; no outro, foi utilizada prótese biológica.

Em todos os casos de mixoma foi possível a ressecção completa do tumor. $\mathrm{O}$ aspecto anatomopatológico foi variado e não foram encontrados sinais de malignidade.

O segundo tumor mais encontrado foi o fibroma em 5 ( $9,6 \%$ dos casos), sendo $3(60 \%)$ em ven- trículo direito (VD); 1 (20\%) em ventrículo esquerdo (VE) e $1(20 \%)$ em ambos os ventrículos. Este último caso ocorreu em uma criança de 2 anos de idade, cujo tumor era intramural e acometia o septo interventricular extensamente, tendo sido ressecado com sucesso.

Tivemos $2(3,84 \%)$ casos de rabdomiossarcoma; 1 em uma criança de 7 anos, operada há 26 anos, acompanhada durante 13 anos, assintomática, após o que perdemos o contato; outro caso foi de um rapaz de 21 anos, sem acompanhamento tardio. Em ambos só foi possível realizar toracotomia exploradora seguida de estudo histopatológico.

Em uma criança com 15 dias de vida o tumor encontrado no ventrículo esquerdo foi o rabdomioma $(1,9 \%)$, diagnóstico feito intraútero por eco-fetal e confirmado, posteriormente, pelo exame anatomopatológico. Realizada a ressecção, foi necessário desinserir e reimplantar o músculo papilar anterior da valva mitral, comprometido pela neoplasia. A criança faleceu no pós-operatório imediato, por insuficiência miocárdica.

Entre os tumores benignos, tivemos $1^{31,33}$ caso de fibroelastoma papilar, localizado em região septal da via de saída do ventrículo esquerdo (VSVE). O diagnóstico pré-operatório era de insuficiência aórtica (IAo) severa e massa tumoral em VSVE. Durante a exploração cirúrgica, o tumor tinha aspecto gelatinoso sugestivo de mixoma, porém o exame anatomapatológico evidenciou um fibroelastoma papilar. Realizada plastia aórtica com implante de duas monocúspides de pericárdio bovino (PB) em posição não coronariana e coronariana direita. Está em evoluçāo clínica com moderada IAo e acompanhamento de 1 ano e 9 meses, sem sinais de recidiva.

Ocorreram 3 (57\%) óbitos imediatos, cujas causas foram: falência de múltiplos órgãos em 1 paciente, complicação cerebral em outro e o último faleceu por insuficiência miocárdia.

No período de 52,1 meses, temos o acompanhamento de $20(40,8 \%)$ pacientes, todos em classe funcional I. Houve apenas 1 caso de recidiva tumoral em paciente portador de mixoma de $\mathrm{AE}$ três anos a $1^{\text {a }}$ intervençâo cirúrgica e no mesmo local.

Reoperado e ressecado o mixoma, não retornou para acompanhamento.

\section{COMENTÁRIOS}

É atribuído a Columbus, em 1559 , o primeiro reconhecimento de um tumor do coração; seguido por Malpighi, que, em 1666, escreveu uma "dissertaçăo de polypo cordis", e Morgagni sobre "Tumores do Coração", em 1762. 
ABDULMASSIH, NETO, C.; SALERNO, P.R.; DINKHUYSEN, J.J.; CHACCUR, P.; ARNONI, A.S.; ZAMORANO, M.M.B.; JATENE A.D.; SOUZA, L.C.B.; PAULISTA, P.P. - Correção cirúrgica dos tumores primários do coraçäo. Rev. Bras. Cir. Cardiovasc.,
7(2):145-152, 1992 .

O primeiro relato sobre o diagnóstico clínico de um tumor primário do coração, um sarcoma, foi realizado em 1934 e o diagnóstico angiográfico do mixoma foi efetuado pela primeira vez em $1951^{16}$.

O grande marco, no entanto, ocorreu em 1968, quando, através da ecocardiografia, foi diagnosticado um mixoma de átrio esquerdo, confirmado na operação ${ }^{16}$, embora Crafoord já houvesse excisado com sucesso um mixoma atrial em $1954{ }^{16}$.

As neoplasias do coraçāo e pericárdio são mais ou menos infreqüentes. A incidência média em necropsia é muito baixa, variando de $0,0001 \%$ a $0,05 \%{ }^{31}$.

Atualmente, o diagnóstico é feito com maior freqüência e a prevalência na cirurgia cardíaca difere entre $0,0013 \%$ e $0,005 \%{ }^{27}$, entre os vários grupos, ocorrendo em $0,09 \%$ nos pacientes operados no Instituto Dante Pazzanese de Cardiologia.

Os mixomas são os tumores benignos mais freqüentes e mais relatados. Alguns ${ }^{\circ}$ observaram a alta incidência de até $88 \%$. Constatamos uma incidência de $82,6 \%$.

A maioria dos mixomas cardíacos ocorre esporadicamente, usualmente em pacientes do sexo feminino, de meia idade, na $5^{\mathrm{a}}$ ou $7^{\mathrm{a}}$ décadas de vida, como um tumor isolado no interior do átrio esquerdo, sem outras alteraçōes ${ }^{24}$. Algumas séries mostram quase igual distribuição entre os sexos ${ }^{18}$.

Em nossa experiência, mais de $53,84 \%$ dos pacientes portadores de mixoma tinham insuficiência cardíaca congestiva. Episódios sincopais são usualmente relatados com mudanças posturais $e$ podem causar disfunção valvar, arritmias cardíacas, ou distúrbios de condução. A obstrução valvar acontece freqüentemente em mixomas pediculares que prolapsam através das valvas atrioventriculares, causando morte súbita.

Embolia por fragmento de tumor tem sido descrita freqüentemente para o cérebro, pulmōes e perifericamente. Esses fragmentos raramente crescem nesses locais ${ }^{33}$. Os mixomas apresentam uma incidência de $24 \%$ de trombo-embolismo ${ }^{21}$, tendo ocorrido em apenas um paciente da nossa série. Cerca de $50 \%$ dos êmbolos envolvem artérias do sistema nervoso central ${ }^{10}$ (embolia da artéria central da retina).

Síncope ou morte súbita acontece em menos de um quarto dos pacientes com mixoma do átrio esquerdo, em um terço com mixoma do átrio direito e ventrículo direito e um quinto com mixoma do ventrículo esquerdo ${ }^{20}$.

Embolias pulmonares ocorrem em $10 \%$ dos casos, provenientes de tumores localizados no lado direito do coraçăo ?.
Arritmias e defeitos de condução podem-se originar de irritação local, isquemia, esclerose ou invasão do sistema de conduçāo ${ }^{32}$.

Sintomas gerais e modificaçōes laboratoriais são mais encontrados em pacientes com mixomas; incluem febre, perda de peso, astenia, mialgia, artralgia, anemia, trombocitopenia, baqueteamento digital, níveis elevados de globulinas, proteína $\mathrm{C}$ - reativa e aumentos dos níveis de eritrossedimentação ${ }^{3}$.

Também em pacientes com mixomas do átrio, anticorpos antimiolena estavam significamente aumentados, quando comparados com controles. Anticorpos antisarcolema foram positivos em $100 \%$ das pontes trivalentes de imunoglobina. Anticorpo antiendotelial do tipo Ig G pode ser encontrado em $86 \%$ dos pacientes com mixoma atrial ${ }^{19}$.

Outros sintomas, embora menos freqüentes, são: hemoptise, dor torácica atípica, ascite, edema periférico e tamponamento cardíaco.

Derrame pericárdio e tamponamento cardíaco sāo freqüentemente descritos nas formas agressivas de mixomas e nos tumores malignos ${ }^{34}$.

Não obstante o reconhecimento do mixoma cardíaco ser uma neoplasia verdadeira, seu potencial maligno tem sido debatido.

MCALLISTER \& FENOGLIO Jr. ${ }^{18}$ chamaram de mixomas cardíacos malignos exemplos de sarcomas com extensas áreas de degeneraçāo mixóide ou múltiplos mixomas benignos. Nós preferimos a denominação para essas formas de "myxoid imitators", proposta por STOUT \& LATTERS ${ }^{30}$. A invasão $e$ transgressão de paredes arteriais, as mudanças de tecido muscular por mixóide têm sido evidências de comportamento "maligno" de alguns mixomas ${ }^{25}$.

A origem multicêntrica do tumor e a ressecçăo primária inadequada são causas estabelecidas de recorrência; a possível transformaçāo ou potencial maligno deve ser considerada quando um tumor recorrente é detectado ${ }^{25}$.

O desenvolvimento de aneurismas micóticos é ocasionado por embolias e o resultado de infiltração de parede arterial por células do mixoma ${ }^{10}$.

Tem sido sugerido que pode ocorrer a transformação maligna do êmbolo mixomatoso, mas a causa dessa transformação não é clara. Uma explicação pode ser a posição "imunológica privilegiada" do cérebro, que permite o tumor crescer mais rapidamente, pois ele é protegido da defesa imunológica do hospedeiro 5 .

A distinção clínica e patológica entre mixoma benigno e agressivo é difícil de prever. A identificação de figuras mitóticas pode auxiliar, mas não é indício histológico de comportamento maligno ou de aparência benigna. 
ABDULMASSIH, NETO, C.; SALERNO, P.R.; DINKHUYSEN, J.J.; CHACCUR, P.; ARNONI, A.S.; ZAMORANO, M.M.B.; JATENE, A.D.; SOUZA, L.C.B.; PAULISTA, P.P. - Correçāo cirúrgica dos tumores primários do coraçäo. Rev. Bras. Cir. Cardiovasc., $7(2): 145-152,1992$.

Além desse comportamento agressivo, a literatura apresenta vários relatos de ocorrência familiar desse tumor, associado a lesōes da pele e outros tumores endócrinos ${ }^{31}$. Também existem as chamadas formas complexas ou sindrômicas, cujas características incluem pacientes jovens, alta incidência em múltiplos locais (inclusive ventrículos) e uma tendência para recorrência.

O primeiro mixoma cardíaco familiar foi descrito por KRAUSE et alii ${ }^{17}$, em 1971. Até o momento, 16 outras famílias têm sido identificadas. Originalmente, pensou-se que a forma familiar era transmitida por um gene autossômico recessivo com influência variável de fatores ambientais. Hoje, mais precisamente, sabe-se ser transmitido através de um traço autossômico dominante, bem documentado em pais e filhos ${ }^{23}$. Tem sido descartada a herança recessiva ligada ao sexo, já que esta entidade patológica pode ser transmitida de pai para a filha. Como se vê, a ligação hereditária não está ainda bem substanciada ${ }^{33}$.

Outra hipótese de transmissão envolve infecção por vírus Coxsakie B4. Estudos com microscopia eletrônica e corando anticorpos imunofluorescentes têm revelado antígeno viral Coxsakie B4 e partículas virais no citoplasma de células estreladas em mixomas ressecados do átrio direito ${ }^{6}$.

A ocorrência de múltiplas e recidivantes lesōes tem sido associada, nāo siomente com a forma familiar, mas também com a forma de síndrome.

Inicialmente, foi descrita por CARNEY ${ }^{7}$ uma forma complexa de mixoma associada com manchas pigmentadas e doença nodular adrenocortial, com hiperatividade glandular.

Várias outras síndromes têm sido descritas.

ATHERTON et alii ${ }^{2}$ usaram o termo "NAME syndrome", que significa nevus, mixoma atrial, neurofibroma mixóide e efélides.

RHODES et alii ${ }^{26}$, descreveram a "LAMB syndrome", constituída de lesōes e lentiginosas da pele, mixoma atrial e "blue nevi". Lesōes da glândula tireóide têm sido infreqüentemente notadas com esse complexo.

Formas familiares e complexas podem ser caracterizadas pela presença de lesōes associadas de pele, fibroadenomixoma mamário, síndrome de Cushing, tumor de hipófise e lesōes de útero e testículo (tumor calcificado das células de Sertoli ${ }^{33}$ ).

Alteraçōes hematológicas incluem anemia hemolítica e trombocitopenia.

Nos últimos anos, o diagnóstico dos tumores cardíacos tem sido grandemente melhorado pela ecocardiografia bidimensional ou transesofágica, tomografia computadorizada e imagem por ressonância magnética.

Como se sabe, nāo é possível determinar, através da ecocardiografia, o tipo histológico. Algumas características podem, no entanto, sugerir malignidade, como uma extensão mediastinal, crescimento rápido do tumor, localizaçāo do lado direito, ou metástases.

A tomografia computadorizada quase sempre permite diferenciar, entre o tecido tumoral e o normal, o que é de grande interesse em tumores infiltrativos, os quais são detectáveis através da ecocardiografia.

A tática cirúrgica depende do tipo, do local e da sintomatologia.

Insistimos em que a retirada cirúrgica do mixoma deve ser feita, sempre que possível, logo após o diagnóstico, pelo alto risco de obstruçäo valvar e embolização sistêmica.

Para evitar recorrência, ressecamos o mixoma com uma área da parede com endocárdio normal e, subseqüentemente, reparamos o defeito criado com sutura direta, ou interposiçāo de retalho de pericárdio bovino, ou outro material.

Temos, também, empregado, além da via atrial esquerda, a via transeptal que, atualmente, é a preferida, porque facilita a ressecção em bloco, nāo deixando fragmentar a massa tumoral, e dá a possibilidade de inspeção de todas as câmaras cardíacas.

Quando o tumor envolve porçōes não responsáveis do miocárdio, especialmente área do septo atrioventricular, a recorrência é mais comum. A eletrocoagulação da base do tumor é recomendada, o que nảo previne a recidiva. Esta pode ser suspeitada mais precocemente pelos níveis elevados de eritrosedimentaçāo, contagem de leucócitos, taxa de hemoglobina, níveis séricos de proteínas e gamaglobulina.

Outro grupo de tumores tratados em menor número foram os fibromas, rabdomionas, rabdomiossarcomas e fibroelastoma papilar.

Os três primeiros sāo mais comuns na infância e adolescência e seu reconhecimento clínico é importante porque muitos deles sāo favoráveis à ressecção cirúrgica.

Os resultados da intervençāo cirúrgica em pacientes com fibromas cardíacos têm sido satisfatórios, atualmente, exceto em pacientes com enorme massa ocupando a maior parte de ambas as cavidades ventriculares, apresentando-se em severa insuficiência cardiaca congestiva, taquicardia ventricular recorrente ou fibrilação no período neonatal ${ }^{36}$. 
ABDULMASSIH, NETO, C.; SALERNO, P.R.; DINKHUYSEN, J.J.; CHACCUR, P.; ARNONI, A.S.; ZAMORANO, M.M.B.; JATENE, A.D.; SOUZA, L.C.B.; PAULISTA, P.P. - Correçāo cirúrgica dos tumores primários do coraçāo. Rev. Bras. Cir. Cardiovasc., 7(2):145-152, 1992.

Excelente sobrevida a longo prazo após completa ressecçāo tem sido relatada por WILLIAMS et alii ${ }^{35}$.

O rabdomioma é o mais freqüente tumor cardíaco do recém-nascido, predominando no sexo masculino na proporçăo de $2: 1$. Na citação de 36 casos, por FENOGLIO Jr. et alii ${ }^{13}, 53 \%$ dos recémnascidos falecem na primeira semana e $78 \%$ antes de completar o primeiro ano de vida. Observam-se as mais variadas formas de manifestaçōes clínicas, desde arritmias cardíacas até quadros de obstrução cavitária, levando rapidamente à morte. Às vezes, podem ser assintomáticos, ou ocorrer regressāo das alteraçōes clínicas, por nāo existir importante comprometimento da função cardíaca.

Esse comportamento clínico e o aspecto microscópico desses tumores sugerem que as células do rabdomioma perdem, durante a evolução, sua capacidade de se dividir. Desse modo, acredita-se que os rabdomiomas cardíacos, por nāo apresentarem desdiferenciaçāo celular, como ocorre com as células neoplásicas, sāo considerados malformaçōes semelhantes a tumores que poderiam ser reconhecidos como hamartomas fetais, e nāo um neoplasma verdadeiro ${ }^{13,22,29}$.

Os rabdomiomas podem se apresentar associados a várias anomalias congênitas, como angiomiolipomas ${ }^{21}$, hamartomas de rins, rim multicístico e quase sempre com esclerose tuberosa na prevalência de $50 \%$ a $86 \%$.

Foi observada uma tendência dos rabdomiomas regredirem na infância e adolescência, desde que haja uma condiçāo clínica favorável ${ }^{29}$.

Já o rabdomiossarcoma, também originário dos miócitos, é o mais freqüente tumor maligno, após o angiossarcoma, e constitui $0,25 \%$ de todos os tumores cardíacos ${ }^{18}$. As cavidades cardíacas direitas sāo as mais afetadas e a disseminaçāo hematogênica sempre ocorre.

Quando o diagnóstico é estabelecido, $30 \%$ a $68 \%$ desses tumores já apresentam metástases, sāo altamente agressivos e a média de sobrevida sem tratamento é de seis meses após ter sido feito o diagnóstico. Somente uma sobrevida mais longa que dois anos tem sido relatada ${ }^{12}$.

A ressecçāo ampla, às vezes, com reconstrução cardíaca é requerida, seguida de quimioterapia e radioterapia.

Em 1985, COOLEY et alii ${ }^{8}$ descreveram um novo procedimento cirúrgico para tratar extensivos tumores que envolvem a parede posterior do coração. Executaram autotransplante após completa excisāo da massa.

Por último, o fibroelastoma papilar, que, na grande maioria, é um tumor das valvas cardíacas, no nosso material foi de origem nāo valvar.

Essas lesōes benignas sāo pequenos tumores derivados de componentes normais do endocárdio, tecido fibroso, fibras elásticas e células musculares lisas ${ }^{11}$.

Quando as valvas atrioventriculares sāo envolvidas, o tumor é mais freqüente no lado atrial, mas, quando sāo as semilunares, nāo há predileçāo por um lado ou por outro.

Na cúspide mitral, o local mais comum de ocorrência é na porção medial, entre o anel e a borda livre. A valva aórtica é a mais acometida, mas sem comprovaçāo estatística significativa.

O tamanho dos tumores varia de $3 \mathrm{~mm}$ a $7 \mathrm{~cm}$ na sua maior dimensão.

Por causa da raridade desses tumores, suas características clínicas ainda nāo são completamente compreendidas. Todavia, é de particular importância que essas lesōes sejam reconhecidas com mais freqüência, para apreciaçāo de seu comportamento e de suas implicaçōes cirúrgicas.

Alguns, ainda, sāo achados acidentais de necropsia, mas recentemente tem havido um inequivoco aumento no diagnóstico em vida ${ }^{14}$.

Têm sido responsáveis por morte súbita, eventos embólicos e falência cardíaca em numerosos e bem documentados casos. Os êmbolos podem originar-se de fragmentos do tumor ou de trombos que podem formar-se em torno do próprio tumor ${ }^{15}$.

O comportamento clínico desses tumores é algo menos agressivo do que os tumores extravalvares, mas, em decorrência do potencial de complicaçōes graves, devem ser tratados cirurgicamente. É recomendada a anticoagulaçāo logo após a ressecçāo.

\section{CONCLUSŌES}

Os tumores benignos, representados, no nosso trabalho, pelos mixomas, podem ser retirados cirurgicamente, com baixa mortalidade.

Os tumores nāo mixomatosos são mais raros, o prognóstico depende da ressecabilidade e da forma clínica com que se apresentam, mas, em geral, os resultados são bons, mesmo após incompleta ressecção.

A nossa experiência com os tumores malignos é limitada e com maus resultados; o diagnóstico e os procedimentos paliativos, associados a quimioterapia e radioterapia, sāo importantes como instrumentos para melhorar e prolongar a vida. 
ABDULMASSIH, NETO, C.; SALERNO, P.R.; DINKHUYSEN, J.J.; CHACCUR, P.; ARNONI, A.S.; ZAMORANO, M.M.B.; JATENE, A.D.; SOUZA, L.C.B.; PAULISTA, P.P. - Correção cirúrgica dos tumores primários do coração. Rev. Bras. Cir. Cardiovasc., 7(2):145-152, 1992.

RBCCV 44205-173

ABDULMASSIH, NETO, C.; SALERNO,P.R.; DINKHUYSEN, J. T.; CHACCUR, P.; ARNONI, A.S.; ZAMORANO,

M.M.B.; JATENE, A.D.; SOUZA, L.C.B.; PAULISTA, P.P. - Surgical correction of primary cardiac tumors.

Rev. Bras. Cir. Cardiovasc., 7 (2): - , 1992

ABSTRACTS: Fifty two patients with primary cardiac tumors were operated on at the Instituto Dante Pazzanese de Cardiologia between 1962 and 1991 . Among the benign neoplasms, 43 were myxomas; the only two malignant tumors were rhabdomyosarcomas. The mean age of the patients was 41 years (range 15 days to 68 years); 33 patients were females and 19 males; $52.6 \%$ had congestive heart failure, $18 \%$ had chest pain or palpitation and $16 \%$ taquiarrhythmias. Complete resection was carried out in 50 patients. The two patients with malignant tumors had only been submitted to biopsy. All patients survived operation, although 3 died in the early postoperative period. One patient with myxoma presented recurrence three years after the innitial surgery and was reoperated on. The follow up data of 20 patients are available, all in NYHA functional class I. Longterm results were excellent in this group.

DESCRIPTORS: heart tumors, surgery.

\section{REFERÊNCIAS BIBLIOGRÁFICAS}

1 ANTUNES, M.J.; VANDERDONCH, K.M.; ANDRADE C.A.; REBELO, S.C. - Primary cardiac leiomyosarcomas. Am. Thorac. Surg., 5:999-1001, 1991.

2 ATHERTON, D.J.; PITCHER, D.W.; WELLS, R.S.; Mac DONALD, D.M. - A syndrome of cutaneous pigmented lesions, myxoid neurofibromas and atrial myxornas: The Name Syndrome. Br. J. Dermatol., 103:421-429, 1980.

3 ATTUM, A.A.; JOHNSON, G.S.; LAUSING, A.M. - Malignant clinical behavior of cardiac myxomas and "myxoid imitators". Ann. Thorac. Surg., 44:217-222, 1987.

4 BISEL; H.F.; WROBLEWSKI, F.; LA DUE, J.S.; Incidence and clinical presentation of cardiac metastasis. Jama, 153:712-715, 1953.

5 BUDZILOVICH, G.; ALEKSIC, S.; GRECO, A. - Malignant cardiac myxona with cerebral metastases. Surg. Neurol., 11:461-470, 1979.

6 BURCH, G.E.; SHEWEY, L.L.; HARB, J.M. - Coxsackie B4 viruses and atrial myxoma. Am. Heart J., 88:634-638, 1974.

7 CARNEY, J.A. - Differences between non-familial and familial cardiac myxoma. An. Surg. Pathol., 9:53$55,1985$.

COOLEY, D.A.; REARDON, M.J.; FRAZIER, O.H.; ANGELINI, P. - Human cardiac explantation and autotransplantation: application in a large cardiac pheochromocytoma. Texas Heart Inst. J., 9:1103-1105, 1985.

9 DEIN, J.R.; FRIST, W.H.; STINSON, E.B. - Primary cardiac neoplasmas: early and late results of surgical treatment in $\mathbf{4 2}$ patients. J. Thorac. Cardiovasc. Surg., 93:502-511, 1987.
10 DeSOUZA, A. L.; MULLER, J.T.; ROBERTSON, J.H. Atrial myxoma: a review of the neurological complications, metastasis and recurrences. $J$. Neurol. Neurosurg. Psychiatry, 41:1119-1123, 1978.

11 EDWARDS, F. H.; HALE, D.; COHEN, A.; THOMPSON, L.; PEZZELLA, VIRMANI, R. - Primary cardiac valve tumors. Ann. Thorac. Surg., 52:1127-1131, 1991.

12 EISENMANN, B.; BAHEREL, B.; BOREISS, P.; JEANBLANC, B.; WARTER, J.; KIENY, R. - Les sarcomes primitifs de l'artère pulmonaire. Ann. Chir. Thorac. Cardiovasc., 16:315-322, 1977.

13 FOWLES, R. E.; MILLER, D.G.; EGBERT, R. M.; FITZGERALD, J. W.; POP, R. L. - Systemic embolization from a mitral valve papillary endocardial fibroma detected by two-dimensional echocardiografy. Am. Heart J., 102:128-130, 1981.

14 FENOGLIO Jr., J. J,; McALLISTER Jr., H. A; FERRANS, V. J. - Cardiac rabdomyoma: clinicopathologic and eletron microscopic study. Am. J. Cardiol., 38:241251, 1976.

15 KARSAKIS, E. J.; O'CONNOR, W.; EARLE, G. - Embolic stroke from cardiac papillary fibroelastomas. Stro$k e, 19: 1171: 1173,1988$.

16 KIRKLIN, J. \& BARRAT-BOYES, B. - Cardiac surgery: morfology, diagnostic criteria, natural history, techniques, results and indication. New York, Wiley Medical Publications, 1986. Cap. 50, p. $1993-1407$.

17 KRAUSE, S.; ADLER, L. N.; REDDY, P. S.; MAGOVERN, G. J. - Intracardiac myxoma in siblings. Chest, 60: 404-406, 1971.

18 MCALLISTER, H.A. \& FENOGLIO Jr., J.J. - Tumors of the cardiovascular system. In: Atlas of tumor pathology. Washington D.C., Armed Forces Institute of Pathology, 1978. Fascículo 15, série 2. 
ABDULMASSIH, NETO, C.; SALERNO, P.R.; DINKHUYSEN, J.J.; CHACCUR, P.; ARNONI, A.S.; ZAMORANO, M.M.B.; JATENE, A.D.; SOUZA, L.C.B.; PAULISTA, P.P. - Correção cirúrgica dos tumores primários do coração. Rev. Bras. Cir. Cardiovasc. 7(2):145-152, 1992.

MAISCH, B. - Immunology of cardiac tumors. Thorac. Cardiovasc. Surg., 38 (Supl. 2):157-163, 1990.

MELLER, J.; TEICHHOLZ, L. E.; PICHARD, A. D. - Left ventricular mixoma: echocardiografic diagnosis and review of the literature. Am. J. Med., 63:816-823, 1977.

MIRALLES, A.; BRACAMONTE, L.; SONCUL, H.; DIAZ DEL CASTILLO, R.; AKTAR, R.; BORS, V.; PAVIE, A.; GRANDJBACKHCH, I.; CABROL, C. - Cardiac tumor: clinical experience and surgical results in 74 patients. Ann. Thorac. Surg, 52:886-895, 1991.

NADAS, A. S. \& ELLISON, C. - Cardiac tumors in infancy. Am. J. Cardiol., 2:363-375, 1968.

POWERS, J. C.; FALKOFF, M.; HEINLE, R. A.; NANDA, N. C.; ONG, L.S.; WEINER, R. S.; BAROLD, S. S. - Familial cardiac mixoma: emphasis on unusual clinical manifestations. J. Thorac. Cardiovasc. Surg. 77:782-788, 1979.

PRICHARD, R.W. - Tumors of the heart: review of the subject and report of 150 cases. Arch. Pathol., 51:98-102, 1951

READ, R. C.; WHITE, H. T.; MURPHY, M. C. - The malignant potentiality of left atrial mixoma. $J$. Thorac. Cardiovasc. Surg., 66:857-867, 1974.

RHODES, A. R.; SILVERMAN, R. A.; HARUST, T. J.; PEREZ-ATAYDE, A. R. - Mucocutaneous lentigines, cardiomucocutaneous mixomas and multiple blue nevi the Lamb syndrome. J. Am. Acort. Dermatol., 10:72-82, 1984
SEZAI, Y. - Tumors of the heart: incidence and clinical importance of cardiac tumors in Japan and operative technique for large left atrial mixomas. Thorac. Cardiovasc. Surg., 38 (Supl. 2):201-204, 1990.

SCHMALTZ, A. A. \& APITZ, J. - Primary heart tumors in infancy and childhood. Cardiology, 67:12-22, 1991.

SOUZA, D. R. S. \& GRECO, O. T. - Tumores cardíacos. Rev. Soc. Cardiol. E.S.P., 1:59-64, 1992.

STOUT, A. P. \& LATTERS, R. - Tumors of the soft tissues. In: Atlas of tumor pathology. Washington D.C., Armed Forces Institute of Pathology, 1967. Fascículo 1, série 2, p. 35-37.

STRAUS, R. \& MERLISS, R. - Primary tumor of the heart. Arch. Pathol., 39:74-78, 1945.

TILLMANNS, H. - Clinical aspects of cardiac tumors. Thorac. Cardiovasc. Surg., 38 (Supl. 2):152-156, 1990.

3 VAN GELDER, M. H.; O'BRIEN, J. D.; STAPLES, D. E.; ALEXANDER, J. A. - Familial cardiac mixoma. Ann. Thorac. Surg., 53:419-424, 1992.

WEINLERY, B. A.; CACES Jr., D. J.; WALLER, B. F.

- Cardiac manifestations of no-cardiac tumors. I: Direct effects. Clin. Cardiol., 12:289-296, 1989.

35

WILLIAMS, D. B., DANIELSON, G. K.; McGOON, D. C.; FELDT, R. H.; EDWARDS, W. D. - Cardiac fibromas: long-term survival after excision. J. Thorac. Cardiovasc. Surg., 84:230-236, 1982.

36 YAMAGUCHI, M.; HOSOKAWA, Y.; MINAMIJI, K. Cardiac fibroma. J. Thorac. Cardiovasc. Surg., 103:140-145, 1992.

\section{Discussāo}

DR. DIVINO PINTO

São Paulo, SP

Agradecemos, inicialmente, a Comissāo Organizadora, pela oportunidade de comentar trabalho de tão alta importância no âmbito da cirurgia cardíaca. A título de colaboração, gostaria de apresentar a nossa estatística dos últimos 10 anos, compreendida por 51 casos de tumores cardíacos, sendo 12 em crianças em que houve predomínio de abdomioma ( 7 casos), seguido de fibroma, mixoma e papiloma, na faixa etária de 40 dias a 7 anos; 8 destes tumores ocorreram em crianças menores de 2 anos. O restante, 39 casos, em adultos com incidência de $80 \%$ de mixomas com comportamento semelhante ao citado pelo autor. Um mixoma de ventrículo direito teve, como único sintoma, T.P.S.V., outro de ventrículo esquerdo, palpitaçōes, o qual recidivou 8 anos após a cirurgia, com sopro cardíaco detectado após síncope (obstrução da via de saída do ventrículo esquerdo). Recentemente, operamos paciente portador de fibroma ocupando grande parte do ventrículo esquerdo comprometendo suas paredes anterior e lateral. Gostaria de chamar atenção para o fato de, não raramente, ser necessária a intervençāo sobre cavidades miocárdicas, de forma a propiciar boa margem de segurança ao procedimento. Como foi comentado, o cirurgiāo deve estar atento e preparado para situaçōes em que possa ser necessária a reconstruçăo da parede. Já os tumores malignos do coraçāo ocorrem em cerca de $25 \%$ dos tumores cardíacos. No trabalho apresentado, apenas 2 casos em 52 eram malignos. Isto representa apenas $3,84 \%$ o que, provavelmente, deve ter ocorrido é que, devido à evolução rápida desses tumores, parte dos pacientes não chega a ter acesso ao tratamento cirúrgico. O tumor maligno mais freqüente é o angiossarcoma. Um destes tumores foi operado no Incór, no qual necessitamos ressecar grande parte do átrio direito e reconstruir a parede com enxerto biológico. Para finalizar, gostaria de lembrar que, atualmente, o exame com a ressonância magnética tem complementado os estudos ecocardiográfico e tomográfico na semiologia desses tumores, com várias citaçōes recentes na 
ABDULMASSIH, NETO, C.; SALERNO, P.R.; DINKHUYSEN, J.J.; CHACCUR, P.; ARNONI, A.S.; ZAMORANO, M.M.B.; JATENE, A.D.; SOUZA, L.C.B.; PAULISTA, P.P. - Correção cirúrgica dos tumores primários do coração. Rev. Bras. Cir. Cardiovasc., $7(2): 145-152,1992$.

literatura mostrando a acurácia desse exame. Aproveito para cumprimentar o Dr. Camilo Abdulmassih Neto e seus colaboradores, pelo trabalho e sua brilhante exposiçāo, cuja apresentaçăo deixou-nos muito pouco a acrescentar. Gostaria de ouvir algum comentário sobre a evoluçāo dos dois pacientes portadores do rabdomiossarcoma, uma vez que a literatura refere uma sobrevida muito curta.

\section{DR. CAMILO}

(Encerrando)

Agradeço os comentários do Dr. Divino, onde apreciamos sua casuística de bons resultados. Concordamos plenamente com o uso da ressonância magnética, a qual, por não ser invasiva e de alta resoluçāo, traz importantes subsídios para o cirurgião. No tocante aos dois casos de rabdomiossarcoma, o primeiro foi em uma criança de 7 anos. Operada há 26 anos, assintomática durante 13 anos e, posteriormente perdemos o contato com a mesma. O caso seguinte era um rapaz de 21 anos, do qual não obtivemos seguimento tardio. Entretanto, sabemos, de literatura, que o resultado tardio de ex-pacientes portadores de rabdomiossarcoma é bastante reservado. Muito obrigado. 